\title{
Distinct Approaches in Managing Adult and Pediatric Trigger Finger
}

\author{
Putu Feryawan Meregawa ${ }^{1}$, John Nolan ${ }^{2}$ \\ ${ }^{1}$ Departement of Orthopaedic and Traumatology, Medical Faculty, Udayana University-Sanglah General \\ Hospital Denpasar, Indonesia \\ ${ }^{2}$ Faculty of Medicine, Udayana University, Denpasar
}

Corresponding Author: Putu Feryawan Meregawa

\begin{abstract}
Background: Trigger finger is the most common disease that causing pain in the adult population. Trigger finger may occur at any finger, which usually occurs in the thumb and ring finger. A1 pulley sheath or metacarpalphalangeal joint. There is a different recommendation toward treatment options for trigger fingers in adults and pediatrics.

Method: This paper is done by reviewing and searching journals with "trigger finger", "treatment", "pediatric trigger finger", and "adult trigger finger" on the search engines. The authors found 45 journals that were suitable for our references.

Outcome: Trigger finger treatments vary as there are many acceptable treatment options. There is a different choice of therapy that should be considered in adult and pediatric trigger fingers. This should be considered to get better treatment outcomes. Cost analysis is also important to choose the most cost-effective treatment for trigger finger patients.

Conclusion: There is no exact consensus recommendation for choosing the exact treatment. pediatric trigger finger is caused by a multifactorial aspect resulting in unpredictable conservative treatment outcomes unlike in adults. The majority of trigger fingers in pediatrics will need surgical procedures while some cases of adult trigger fingers may resolve naturally. Further large studies are needed to develop a treatment algorithm in these two age groups that trigger fingers.
\end{abstract}

Keywords: adult trigger finger, trigger finger, pediatric trigger finger, treatment

\section{INTRODUCTION}

Trigger finger is a usual circumstance that might create a serious disturbance in quality of life, including functional impairment and hand pain. ${ }^{1}$ Stenosing tenosynovitis or trigger finger is the most common cause of wrist pain among adults, which predisposing women 6 times more compared to men. In the general population, trigger finger affects approximately $2-3 \%$ in prevalence, besides, around $10 \%$ of the patients are predisposed to diabetes. ${ }^{2}$ Most common age which is found with trigger finger is distributed in 2 different age groups, in patients below the age of 8 years old and other aged 40 to 60 years which mean trigger finger can affect adult and pediatric. Trigger finger itself is known as an idiopathic disease, however, repetitive usage of the related hand is thought to be one of the following factors toward the disease. ${ }^{2,3}$ Trigger finger may occur at any finger, which usually occurs in thumb and ring finger. A1 pulley sheath or metacarpal-phalangeal (MCP) joint is the most classically involved. Not only does the proximal portion of the tendon sheath, but also occur at the proximal interphalangeal joint and the distal interphalangeal joint (A2 and A 3$)^{4}{ }^{4}$

The usual complaint that is mostly received by clinicians is pain. Patients feel pain and problem with the locking digits during either flexion and extension. ${ }^{1,3}$ This functional impairment in flexion and tendon mostly cause more problem in extension as 
the flexion strength is much stronger. ${ }^{2}$ An atraumatic catching and locking of the affected finger are usually reported with additional painless snapping. The cause of trigger finger itself is multifactorial, starting from comorbid diseases such as gout, diabetes, thyroid diseases, rheumatoid arthritis, amyloidosis, and carpal tunnel syndrome. ${ }^{5}$ The etiology from the 2 age group is different as in children, the etiology embrace with a developmental problem correlated with incompatibility of the width of the flexor pollicis longus (FPL) tendon of the thumb with the sheath. ${ }^{5,6}$

The treatment options for trigger fingers vary including non-surgical and surgical treatment. ${ }^{1-4}$ The non-surgical or conservative treatments may include splinting to immobilize the joint affected, NSAIDs usage for controlling pain, and corticosteroid injection which the majority are given directly into the sheath. ${ }^{7,8}$ The last option that can be chosen is surgical or operative treatment. This can be done by two methods, by percutaneous release or the open one. Surgical treatment is exceptionally successful and thought to be the answer to a failure in conservative treatment in resolving pain and symptoms. ${ }^{9}$

Trigger fingers can be found in all populations, however, there are quite distinctive things that clinicians should know as we speak about the trigger finger in adults and pediatrics. The 2 age groups should be receiving different treatment alongside the different usual location of the pulley problem. There is a different recommendation toward the treatment options as these decisions put up to the dissimilar outcome. ${ }^{10}$ Clinicians need to know the exact treatment that the patients with trigger

\section{RESULT AND DISCUSSION \\ About trigger finger}

Trigger fingers or stenosing tenosynovitis is the most common problem that causing hand pain. The problem is occurring because of the mismatched size among the flexor tendon and the pulley system of the finger. ${ }^{4,5,11}$ The flexor sheath plays important role in the process of the trigger finger, it is enveloping the flexor digitorum superficialis (FDS) and flexor digitorum profundus (FDP). In these tendons, five annular pulleys and three cruciform pulleys are located which expedite smoother gliding of both tendons to complete finger flexion. A1 pulley, which arises from the palmar plate and overlies the membranous sheath in the MCP joint, is the pulley that is mostly acquired with trigger finger condition. ${ }^{11}$

Repetitive friction of the tendon and pulley is thought to be the etiology of the trigger finger. In adult patients, histological analysis showed that the tissue biopsy consists of fibrocartilaginous metaplasia with additional positive S-100 protein staining which is usually settled in chondrocytes. $^{12}$ People with metabolic diseases including hypothyroidism, diabetes mellitus, and less frequently in gout and mucopolysaccharidosis tend to have a higher risk of developing trigger fingers. ${ }^{13,14}$ The chance of developing a trigger finger is also higher in patients with various rheumatoid conditions such as psoriatic arthritis, rheumatoid arthritis, and, pigmented villonodular synovitis. ${ }^{13,15}$ Not only do rheumatoid conditions but also local disorders including carpal tunnel syndrome, De Quervain's disease, and Dupuytren's disease show a significantly higher incidence of trigger finger. ${ }^{13,15,16}$

\section{$\underline{\text { Adult \& Pediatric Trigger Finger }}$}

Stenosing flexor tenosynovitis commonly attenuates the situation described by clicking, catching, and locking the finger. An epidemiologic study showed that there is a $2.6 \%$ lifetime risk to develop trigger fingers as a higher incidence in some systemic diseases, including diabetes mellitus and inflammatory arthritis. ${ }^{17}$ By doing ultrasound clinicians may notice a significantly thicker and stiffer A1 pulley than normal controls. The diagnosis trigger finger is quite simple, clinicians may establish the diagnosis judged by the clinical 
symptoms. Naturally, the progression of the trigger finger is starting from a painless clicking. These signs are usually followed by pain in triggering or flexing the finger and locking the digit. The complaints may also include radiating pain to the forearm. Further imaging and laboratory diagnostics are not necessary. 2,18

Ultrasound is useful to assess the thickening and the affected sheath compared to the control/normal sheath found to be as meticulous as the manual measurement during a surgical procedure. Ultrasound may help to provide a correlation between the degree of thickening with the severity of the disease. ${ }^{19}$ In terms of grading, there are no universal grading or gold standard grading for trigger finger. The grading system that is commonly used is the Quinnell grading system. $^{20}$

Several differential diagnoses can be concluded toward the condition of patients with trigger fingers. Some of them are the condition that can lead to pain, lock, and inflammation in the MCP joint along with dislocation and fracture. In various cases, a nodule at the flexor tendon is usually misjudged by the clinicians as a bone mass. Fracture diagnosis can easily be eliminated with a physical examination, especially the patient did not have a trauma history. ${ }^{6}$

Table 1. Quinnell grading system for trigger finger patients. (Data from Quinnell R. Conservative management of trigger finger. Practitioner 1980;224:187-90)

Quinnell grading system

\begin{tabular}{|l|l|}
\hline \multicolumn{2}{|c|}{ Quinnell grading system } \\
\hline Grade 0 & Flexion pain with no mechanical symptoms \\
\hline Grade 1 & Uneven finger movements without triggering \\
\hline Grade 2 & The triggering finger is actively correctable \\
\hline Grade 3 & Commonly passively correctable \\
\hline Grade 4 & Locking, uncorrectable, fixed contracture \\
\hline
\end{tabular}

Dupuytren contracture, diabetic cheiroarthropathy, tenosynovitis, infectious tenosynovitis, rheumatoid arthritis, diabetic cheiroarthropathy, osteoarthritis, and crystalline arthropathy are often listed as the differential diagnosis as those diseases show pain and finger locking complaints in adults. $^{2,21}$ Whereas in children, deformity associated with trigger thumb can be misjudged as cerebral palsy or arthrogryposis. These conditions are believed to play a major role in causing disproportionate alteration of connective tissues of the hand. To differentiate these akin conditions, clinicians need to assess other abnormalities apart from the digits of the hand. ${ }^{6}$

\section{Management of adult trigger finger}

Trigger finger treatments are varied. Distinctive clinical intervention can be used to treat this condition including conservative and surgical treatment. Trigger finger option of treatment shows many various acceptable algorithms. Conservative treatment can be applied initially, modification of activities, and joint immobilization with a custommade splint for instance. ${ }^{22,23}$ Splinting helps to avert friction caused by the flexor tendon that moves through the A1 pulley affected in advance of inflammation resolution. Hence, the first treatment that can be considered is by using joint splints considering its positive results ranging from $50 \%$ to $93 \%$. $^{22,24}$

Corticosteroid injection may also be administered as it is a quite effective firstline treatment for the reason of its low morbidity. The efficacy of steroid injection ranging from $67 \%$ to $90 \%$, additionally, it is safe. Also, the administration of the injection is quite easy and a cost-effective strategy. ${ }^{25,26}$ The techniques most commonly used are the mid axial and palmar needle trajectories.

Although the local injection of steroids is a promising first-line treatment, many of the patients also achieve failure in these treatment outcomes. Some also develop a recurrence of trigger fingers. Patients with long-term onset of the symptoms (>6 months) are not in favor to have triggering resolution. ${ }^{27}$ Better shortterm effect ( 6 weeks of administration) is shown by the administration of triamcinolone (non-water-soluble steroid) compared to dexamethasone (water-soluble medication). ${ }^{28}$ In some cases, minor or major complications may occur. Minor complications related to steroid injections 
are skin depigmentation and fat necrosis. ${ }^{29}$ Whereas major complications are rare however, some studies reported the incidences, including tendon attrition and rupture. In diabetic patients, more awareness is required by cause of local steroid effect in the fluctuation of blood glucose (last up to 5 days). ${ }^{30}$

Surgical treatment is the best choice if conservative treatments are failed including open release and percutaneous release. The gold standard of treating trigger fingers is an open release of the A1 pulley. Incisions are done off the A1 pulley of the involved digit in longitudinal, oblique, or transverse openings. The open release is effective in recovering the motion of the joint along with its minimal risk of infection, neurovascular injury, or recurrent trigger finger. ${ }^{29}$ The timing of the surgical procedure is still controversial however, studies showed that the surgical approach should be considered following a failure in single or multiple corticosteroid injections. The open release aims to do complete sectioning of the A1 pulley. Complication rates in general of open release surgery are marginally greater than percutaneous release, starting from stiffness, infection, reflex sympathetic dystrophy, flexor tendon bowstringing, incision pain nerve transection, flexion deformity, and recurrence $(3 \%){ }^{31}$

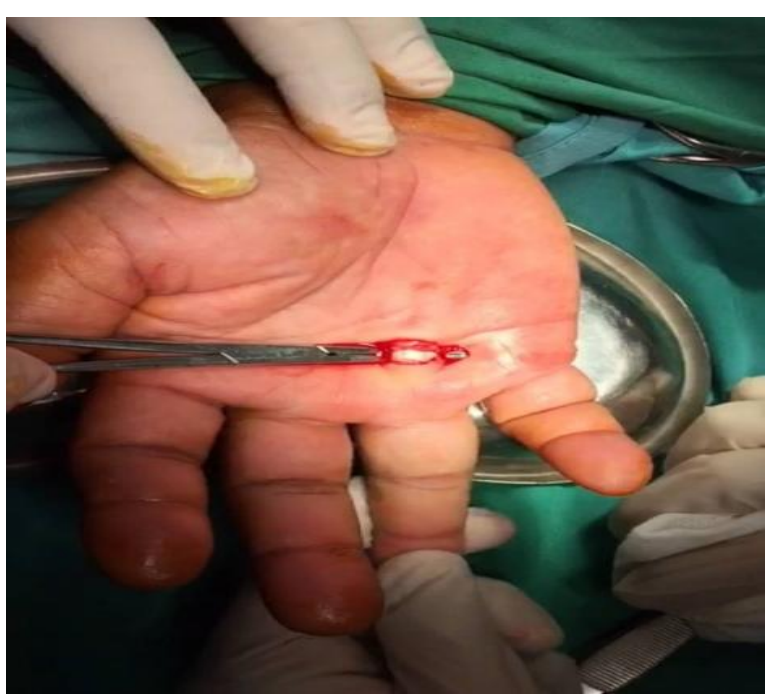

Figure 1. After the pulley released, the flexor tendon can be picked up outside the wound.
The percutaneous release was first introduced in 1958 by Lorthioir. The procedure is somewhat simple with a smooth tenotomy following an aseptic procedure. $^{32}$ This technique is done under local anesthesia, the MCP joint is set hyperextended with the palm facing up. The position helps elongate the pulley and to keep the neurovascular structures in the dorsal. Pain management that is usually applied is ethyl chloride spray with lidocaine injection at the site of incisions. Then, the needle is inserted directly into the A1 pulley before being swept to wedge the pulley proximal and distal to the injection site. There is a risk of digital nerve and artery injury nonetheless, the procedure has beyond $90 \%$ success rates. ${ }^{31,33}$

\section{Treatment of pediatric trigger finger}

The treatment of the pediatric trigger finger can be initially treated with nonsurgical treatment. Trigger finger in pediatric seems more complex and multifactorial. Trigger thumb in pediatric has a similar choice of treatment with adult trigger finger. However, the trigger finger in pediatric is usually affected by other pathology and factors besides the anatomical abnormalities. ${ }^{34,35}$ Trigger finger in pediatric has been highly linked with congenital metabolic and inflammatory disorders such as Down syndrome, EhlersDanlos syndrome, juvenile rheumatoid arthritis, mucopolysaccharidosis, and central nervous system disorders such as delayed motor development, even though that the majority of the cases are idiopathic. ${ }^{36-39}$

Until now, many studies are discussing the best treatment needed for pediatric cases. In pediatric trigger thumb, based on several studies, it resolves naturally in several years. Some studies reported a 5-year follow-up of trigger thumb in pediatric showed improvement in most of the patients despite the failure in pediatric with bilateral trigger thumbs. ${ }^{40,41}$ Surgical procedure in pediatric trigger thumb is not always a necessity as the symptoms will alleviate spontaneously in a few years 
ahead. Majority of patients that demonstrate resolution did not report any recurrences. ${ }^{42}$ For pediatric in trigger thumb, a percutaneous release is not frequently done. A recent report showed that among 15 patients with trigger thumbs, $80 \%$ of the patients were complicated with flexor tendon lacerations. Clinicians should be aware of this method in pediatrics as there is only a slight mean distance of $2.5 \mathrm{~mm}$ between the needle in the percutaneous release procedure and the radial digital nerve. $^{43}$

Trigger finger in pediatric is relatively distinct within the adult. Multifactorial aspects behind the etiology of the condition resulting in unpredictable outcomes of conservative treatments. Until now, surgical intervention is highly recommended for a better clinical outcome. $^{35}$ Besides the A1 pulley release, appendage procedure of A3 pulley release, and/or FDS slip resection, help to alleviate disease probability of recurrence and to increase the chance of symptom resolution. Surgeons should also take a close look at the A 2 or A3 pulley if there is a constriction condition. Over-release of the A2 pulley should be given extra care as it may form to bowstringing of the flexor tendons. Following the procedure, the hand and forearm cast is applied to immobilize the hand until the wounds are completely recovered. ${ }^{6,35}$

\section{Cost Analysis}

The cost of treatment is one of the main issues in facing patients with trigger fingers. Because of that, there is no exact consensus recommendation regarding the choice of treatment for trigger fingers, there are many choices of acceptable treatments. The study conducted by Luther and colleagues compared 4 different treatments of choice for diabetes patients with trigger fingers. $^{44}$ They are one corticosteroid injection before pursued by surgical release, two corticosteroid injections followed by surgical release, immediate surgical release in an OR, and immediate surgical release in a clinic. The study reported that immediate surgical release was the most benefit for the patient with diabetes. They also found that corticosteroid injections were less efficacious, most of them were still needing surgery even after injections. Fluctuation in blood glucose was also considered as a disadvantage in using corticosteroid injections. ${ }^{44,45}$

In the general population, Kerrigan and colleagues did a cost analysis study that showed that corticosteroid injection followed by a second injection for failures or recurrence, before doing a definitive surgery (if needed) is the most cost-effective treatment. ${ }^{45}$

\section{Summary}

Trigger finger is the most common condition that causing hand pain in adults. Trigger fingers may also be acquired by pediatrics. Up to now, there is no exact consensus recommendation for choosing the exact treatment. To be noted, pediatric trigger thumb and finger are quite distinct from the adult trigger finger. Trigger thumb in pediatric is quite similar to the adult trigger finger, while pediatrics trigger finger is caused by a multifactorial aspect and more complex. This results in unpredictable outcomes for conservative treatments. Pediatric trigger thumb may resolve itself with conservative treatments while most of the trigger fingers in pediatric will need a surgical procedure to gain the perfect outcomes. Many studies are needed to develop more about the information of trigger fingers. Large studies will be needed later to develop the preferred algorithm in deciding the best treatment for trigger finger, whether in adult and pediatric.

\section{Acknowledgement: None}

Conflict of Interest: None

Source of Funding: None 


\section{REFERENCES}

1. Parks E. The hand, wrist, and elbow. In: Practical Office Orthopedics. New York, NY: McGraw-Hill Education; 2017.

2. Makkouk AH, Oetgen ME, Swigart CR, Dodds SD. Trigger finger: etiology, evaluation, and treatment. Current Reviews in Musculoskeletal Medicine. 2008 Jun 1;1(2):92-6.

3. Young Kim J, Jin Choi G, Mo Kang D. Clinical significance of proximal interphalangeal joint pain in patients with trigger fingers. Journal of Hand Surgery (European Volume). 2019 May;44(4):37984.

4. Strigelli V, Mingarelli L, Fioravanti G, Merendi G, Merolli A, Fanfani F, Rocchi L. Open Surgery for Trigger Finger Required Combined a1-a2 Pulley Release. A Retrospective Study on 1305 Case. Techniques in hand \& upper extremity surgery. 2019 Sep 1;23(3):115-21.

5. Jeanmonod R, Waseem $M$. Trigger Finger. Treasure Island, FL: StatPearls Publishing; 2017.

6. Schaverien MV, Godwin Y. Paediatric trigger finger: Literature review and management algorithm. Journal of Plastic, Reconstructive \& Aesthetic Surgery. 2011 May 1;64(5):623-31..

7. Leow MQ, Zheng Q, Shi L, Tay SC, Chan ES. Non- steroidal anti- inflammatory drugs (NSAIDS) for trigger finger. The Cochrane Database of Systematic Reviews. 2017 Sep;2017(9).

8. Shakeel H, Ahmad TS. Steroid injection versus NSAID injection for trigger finger: a comparative study of early outcomes. The Journal of hand surgery. $2012 \mathrm{Jul}$ 1;37(7):1319-23.

9. Sato ES, Gomes dos Santos JB, Belloti JC, Albertoni WM, Faloppa F. Treatment of trigger finger: randomized clinical trial comparing the methods of corticosteroid injection, percutaneous release and open surgery. Rheumatology. 2012 Jan 1;51(1):93-9.

10. Giugale JM, Fowler JR. Trigger finger: adult and pediatric treatment strategies. Orthopedic Clinics. 2015 Oct 1;46(4):5619.
11. Xie P, Zhang QH, Zheng GZ, Liu DZ, Miao HG, Zhang WF, Ye JF, Du SX, Li XD. Stenosing tenosynovitis. Der Orthopäde. 2019 Mar 15;48(3):202-6.

12. Sbernardori MC, Bandiera P. Histopathology of the A1 pulley in adult trigger fingers. Journal of Hand Surgery (European Volume). 2007 Oct;32(5):5569.

13. Cakmak F, Wolf MB, Bruckner T, Hahn $\mathrm{P}$, Unglaub F. Follow-up investigation of open trigger digit release. Archives of orthopaedic and trauma surgery. 2012 May 1;132(5):685-91.

14. Tallia AF, Cardone DA. Diagnostic and therapeutic injection of the wrist and hand region. American family physician. 2003 Feb 15;67(4):745-50.

15. Flatt AE. Notta's nodules and trigger digits. InBaylor University Medical Center Proceedings 2007 Apr 1 (Vol. 20, No. 2, pp. 143-145). Taylor \& Francis.

16. Rottgers SA, Lewis D, Wollstein RA. Concomitant presentation of carpal tunnel syndrome and trigger finger. Journal of brachial plexus and peripheral nerve injury. 2009 Dec;4(1):1-4. Rottgers SA, Lewis D, Wollstein RA. Concomitant presentation of carpal tunnel syndrome and trigger finger. Journal of brachial plexus and peripheral nerve injury. 2009 Dec;4(1):1-4.

17. Tung WL, Kuo LC, Lai KY, Jou IM, Sun YN, Su FC. Quantitative evidence of kinematics and functional differences in different graded trigger fingers. Clinical Biomechanics. 2010 Jul 1;25(6):535-40.

18. Katzman BM, Steinberg DR, Bozentka DJ, Cain E, Caligiuri DA, Geller J. Utility of obtaining radiographs in patients with trigger finger. American journal of orthopedics (Belle Mead, NJ). 1999 Dec;28(12):703-5.

19. Guerini H, Pessis E, Theumann N, Le Quintrec JS, Campagna R, Chevrot A, Feydy A, Drapé JL. Sonographic appearance of trigger fingers. Journal of Ultrasound in Medicine. 2008 Oct;27(10): 1407-13.

20. Quinnell RC. Conservative management of trigger finger. The Practitioner. 1980;224:187-90. 
21. Blazar P, Aggarwal R. Trigger fi nger (stenosing flexor tenosynovitis). www.uptodate.com/contents/trigger-fi nger-stenosingflexor-tenosynovitis.

22. Colbourn J, Heath N, Manary S, Pacifico D. Effectiveness of splinting for the treatment of trigger finger. Journal of hand therapy. 2008 Oct 1;21(4):336-43.

23. Sempowski IP. The management of the occasional trigger finger. Canadian Journal of Rural Medicine. 2008 Jul 1;13(3):136.

24. Tarbhai K, Hannah S, von Schroeder HP. Trigger finger treatment: a comparison of 2 splint designs. The Journal of hand surgery. 2012 Feb 1;37(2):243-9.

25. Dala-Ali BM, Nakhdjevani A, Lloyd MA, Schreuder FB. The efficacy of steroid injection in the treatment of trigger finger. Clinics in Orthopedic Surgery. 2012 Dec 1;4(4):263-8.

26. Sato ES, Gomes dos Santos JB, Belloti JC, Albertoni WM, Faloppa F. Treatment of trigger finger: randomized clinical trial comparing the methods of corticosteroid injection, percutaneous release and open surgery. Rheumatology. 2012 Jan 1;51(1):93-9.

27. Newport ML, Lane LB, Stuchin SA. Treatment of trigger finger by steroid injection. J Hand Surg Am 1990;15(5):748-50.

28. Ring D, Lozano-Calderón S, Shin R, Bastian P, Mudgal C, Jupiter J. A prospective randomized controlled trial of injection of dexamethasone versus triamcinolone for idiopathic trigger finger. The Journal of hand surgery. $2008 \mathrm{Apr}$ 1;33(4):516-22.

29. Ryzewicz M, Wolf JM. Trigger digits: principles, management, and complications. The Journal of hand surgery. 2006 Jan 1;31(1):135-46.

30. Wang AA, Hutchinson DT. The effect of corticosteroid injection for trigger finger on blood glucose level in diabetic patients. The Journal of hand surgery. $2006 \mathrm{Jul}$ 1;31(6):979-81.

31. Green D, Hotchkiss R, Pederson W, et al. Tenosynovitis. In: Green's operative hand surgery. 5th ed. London: Churchill Livingstone; 2005.
32. Lorthioir J. Surgical treatment of triggerfinger by a subcutaneous method. J Bone Joint Surg Am 1958;40:793-5.

33. Dormans JP, Squillante R, Sharf H. Acute neurovascular complications with supracondylar humerus fractures in children. The Journal of hand surgery. 1995 Jan 1;20(1):1-4.

34. Shiozawa R, Uchiyama S, Sugimoto Y, Ikegami S, Iwasaki $\mathrm{N}$, Kato $\mathrm{H}$. Comparison of splinting versus nonsplinting in the treatment of pediatric trigger finger. The Journal of hand surgery. 2012 Jun 1;37(6):1211-6.

35. Bae DS, Sodha S, Waters PM. Surgical treatment of the pediatric trigger finger. The Journal of hand surgery. 2007 Sep 1;32(7):1043-7.

36. Cheung JP, Fung BK, Mak KC, Leung $\mathrm{KH}$. Multiple triggering in a girl with Ehlers-Danlos syndrome: case report. The Journal of hand surgery. 2010 Oct 1;35(10):1675-7.

37. Afshar A. Bilateral carpal tunnel syndrome and multiple trigger fingers in a child with mucolipidosis Type III disease. Indian Journal of Plastic Surgery: Official Publication of the Association of Plastic Surgeons of India. 2011 Sep;44(3):517.

38. Cardon LJ, Ezaki M, Carter PR. Trigger finger in children. The Journal of hand surgery. 1999 Nov 1;24(6):1156-61.

39. Tordai P, Engkvist O. Trigger fingers in children. The Journal of hand surgery. 1999 Nov 1;24(6):1162-5.

40. Jung HJ, Lee JS, Song KS, Yang JJ. Conservative treatment of pediatric trigger thumb: follow-up for over 4 years. Journal of Hand Surgery (European Volume). 2012 Mar;37(3):220-4.

41. Baek GH, Lee HJ. The natural history of pediatric trigger thumb: a study with a minimum of five years follow-up. Clinics in orthopedic surgery. 2011 Jun 1;3(2):157-60.

42. Koh S, Horii E, Hattori T, Hiroishi M, Otsuka J. Pediatric trigger thumb with locked interphalangeal joint: can observation or splinting be a treatment option? Journal of Pediatric Orthopaedics. 2012 Oct 1;32(7):719-21. 
Putu Feryawan Meregawa et.al. Distinct approaches in managing adult and pediatric trigger finger.

43. Masquijo JJ, Ferreyra A, Lanfranchi L, Torres-Gomez A, Allende V. Percutaneous trigger thumb release in children: neither effective nor safe. Journal of Pediatric Orthopaedics. 2014 Jul 1;34(5):534-6.

44. Luther GA, Murthy P, Blazar PE. Cost of immediate surgery versus non-operative treatment for trigger finger in diabetic patients. The Journal of Hand Surgery. 2016 Nov 1;41(11):1056-63.
45. Kerrigan CL, Stanwix MG. Using evidence to minimize the cost of trigger finger care. The Journal of hand surgery. 2009 Jul 1;34(6):997-1005.

How to cite this article: Meregawa PF, Nolan J. Distinct approaches in managing adult and pediatric trigger finger. International Journal of Science \& Healthcare Research. 2021; 6(2): 28-35. DOI: https://doi.org/10.52403/ ijshr.20210406 